\title{
Metodología de diseño, observación y cálculo de redes geodésicas interiores en túneles de ferrocarril de alta velocidad
}

\section{Methodology for designing, observing and computing of underground geodetic networks of large tunnels for high-speed railways}

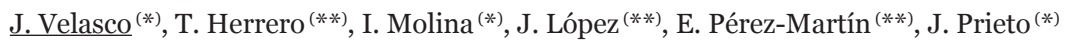

\section{RESUMEN}

El guiado de las Tuneladoras durante su avance debe apoyarse en los puntos determinados inmediatamente detrás de ellas. Conseguir las precisiones requeridas presentaría algunas dificultades en condiciones normales con respecto al exterior, pero en el interior de un túnel se plantean ciertos factores que ensombrecen el panorama. El más importante y difícil de tratar es el de la refracción lateral.

El diseño de redes interiores es uno de los principales problemas, desde el punto de vista geodésico y topográfico, el cual tiene unas características tales que todos los textos de topografía de precisión aconsejan evitar. Con estas redes se va guiando la tuneladora y en ningún momento dicha red vuelve a tener comprobación sobre otros puntos de control. A medida que la red va avanzando, las precisiones obtenidas de sus coordenadas van empeorando de forma exponencial. Este trabajo establece una metodología para el diseño de redes planimétricas interiores.

Palabras clave: Ferrocarriles; túneles; geodesia; redes geodésicas; simulación.

\section{ABSTRACT}

The guiding of TBMs (Tunnel Boring Machines) along their advance must be based on points placed immediately behind them. Achieving good accuracies results would present some difficulties for normal exterior conditions, but inside a tunnel certain factors clouding the outlook arise. The most important and difficult factor to deal with, has to do with lateral refraction.

Internal geodetic networks design is one of the more challenging problems from the geodetic and topographic point of view, as the bibliographical review dealing with this subject, advises to avoid this practice. This network allows only guiding the TBM, so that it is not used for further checking or quality control. As the network progresses, the reached coordinate accuracies deteriorate exponentially. This work establishes a methodology for the design of internal horizontal networks.

Keywords: Railways; tunnels; geodesy; geodetic networks; simulation.

(*) ETSI en Topografía, Geodesia y Cartografía - Universidad Politécnica de Madrid (España).

(**) EUIT Agrícola - Universidad Politécnica de Madrid (España).

Persona de contacto/Corresponding author: jesus.velasco@upm.es (J. Velasco)

Cómo citar este artículo/Citation: Velasco, J., Herrero, T., Molina, I., López, J., Pérez-Martín, E., Prieto, J. (2015). Metodología de diseño, observación y cálculo de redes geodésicas interiores en túneles de ferrocarril de alta velocidad. Informes de la Construcción, 67(538): e076, doi: http://dx.doi.org/10.3989/ic.13.172.

Licencia/License: Salvo indicación contraria, todos los contenidos de la edición electrónica de Informes de la Construcción se distribuyen bajo una licencia de uso y distribución Creative Commons Reconocimiento no Comercial 3.o. España (cc-by-nc). 


\section{INTRODUCCIÓN}

Los vértices de las redes interiores de los túneles se han de referir a la infraestructura señalizada en cada boca mediante, al menos, tres hitos, cuya posición es conocida (red exterior) (1), para ello se debe haber un enlace geométrico mediante un itinerario formado por una sucesión de estaciones unidas por ejes geométricamente definidas a través de sus ángulos y sus distancias (2), los cuales se pueden clasificar en cerrados o encuadrados (3). En el caso de los túneles los itinerarios (poligonales), por lo general, suelen ser cerrados, pero en cualquier caso siempre es preciso disponer de una comprobación a partir de los datos de observación.

Podemos en función de la distribución de los vértices de las redes interiores, hacer una división general (4) en:

- Poligonal en zig-zag: de ésta forma evitamos las observaciones paralelas a los hastiales y minimizamos el error de refracción lateral.

- Poligonales por el eje: Las visuales por el centro del túnel disminuyen el efecto de la refracción lateral, pero llevar una poligonal por el eje es prácticamente imposible.

- Triangulación: se subdivide el túnel en cuadriláteros haciendo las observaciones de forma independiente y compensando mediante el lado común entre polígonos.

Como ejemplos de diseño de una red, decir que en el Canal de la Mancha en la parte inglesa consistió en dos poligonales cruzadas independientes en zig-zag y lados con longitud 150 metros (5). El método elegido en el lado francés consistió en observar los módulos de 375 metros en túnel de servicio. En los túneles de Hallandsas (Suecia) con longitudes de $9 \mathrm{~km}$, el método elegido para la realización de las redes interiores es una combinación de poligonales en zig-zag con ejes de 150 metros añadiendo una estación libre entre cada par de vértices por el centro del túnel (6).

\section{METODOLOGÍA}

Esta metodología comprende el diseño, simulación, observación de redes topográficas planimétricas interiores para la construcción de túneles de gran longitud para ferrocarriles de alta velocidad. Para ello es necesaria una instrumentación adecuada. Estas cuestiones han sido abordadas en los siguientes apartados y ha sido ensayada en la construcción de varios túneles, entre ellos Guadarrama y Pajares. En este estudio vamos a aplicar la metodología a los túneles TAV de Pajares.

\subsection{Instrumentación}

La instrumentación necesaria para la observación de redes planimétricas interiores es la siguiente: instrumentos topográficos-geodésicos de goniometría, distanciometría, giroteodolito y material auxiliar.

\subsubsection{Instrumentos topográficos y geodésicos}

\subsubsection{Estación total}

Para la observación de las redes interiores así como su enlace con las redes exteriores se deben utilizar estaciones totales para mediciones de ángulos y distancias robotizadas con seguimiento de prisma, puntería automática, secuencia de observación programable y registro electrónico de datos. En los túneles de Pajares se han utilizado estaciones totales con precisiones nominales comprendidas entre 1 y 2 segundos centesimales, con distanciómetro de infrarrojos de $\pm(1 \mathrm{~mm}$ $+1 \mathrm{ppm})$.

\subsubsection{Giroteodolito}

La calidad de las redes en el interior de los túneles de gran longitud depende del uso adecuado del giroscopio. En Geodesia y Topografía se utiliza en forma de teodolito giroscópico o giroteodolito. Las medidas de un giroteodolito son equivalentes a determinar acimutes astronómicos, puesto que se orienta siempre al norte geográfico. En (7) se realiza un estudio sobre las aplicaciones del giroteodolito en túneles y en (8) se analiza el modo de calibración del giróscopo. El principio del giroscopio se basa en la reacción del instrumento ante las interferencias externas afectado por el giro terrestre con oscilaciones en el sentido del eje de giro de la interferencia (9). En túneles de Pajares se emplea un giroteodolito compuesto de un giroscopio y una ETR (Estación Total Robotizada de seguimiento automático, con una precisión nominal máxima de $\pm 10^{\mathrm{cc}}$ y registro interno de observaciones (10) (ver Figura 1). Este instrumento permite una búsqueda automatizada del norte giroscópico, pudiendo hacer las punterías a referencia también de forma automática. Este funcionamiento impersonal elimina los errores accidentales subjetivos de seguimiento y puntería, ofreciendo la precisión más alta obtenible con un sistema giroscópico mecánico (11).

Se diseñó un acoplamiento para el giroteodolito que permite estos estacionamientos, ofreciendo la debida precisión de centrado y con la necesaria robustez para garantizar la estabilidad y ausencia de vibraciones durante el funcionamiento del giroscopio (12). En la Figura 1 se puede ver el giroteodolito situado sobre dicho acoplamiento.

\subsection{Constante del giroteodolito}

La constante del giroteodolito no es un ajuste sino una característica mecánica. Es la diferencia entre el norte giroscópico y el norte geográfico o rotacional terrestre. Sus valores se establecen en las redes de boca, en los lados exteriores orientados mediante, en principio, observaciones astronómicas a la estrella Polar para poder determinar el acimut astronómico (13). La diferencia entre la lectura del giroteodolito y el acimut astronómico nos da la constante $K$. Una vez determinado el acimut hay que realizar las correcciones por movimiento del polo, altitud del punto visado y curvatura de la plomada (14) pues es un acimut astronómico instantáneo referido a la superficie geopotencial del punto de observación.

El problema que nos encontramos es que las observaciones astronómicas, como es lógico, no pueden realizarse en el interior de los túneles, por lo que en ellos tendremos acimutes geodésicos. Para el paso de acimut astronómico a geodésico se necesita hacer fundamentalmente: correcciones instrumentales y corrección por desviación de la vertical. La influencia de las irregularidades del campo gravitatorio terrestre (desviación de la vertical) debe ser tenida en cuenta en regiones donde el valor de dicha desviación puede ser mayor que la precisión del giroteodolito. Para calcular dicha com- 


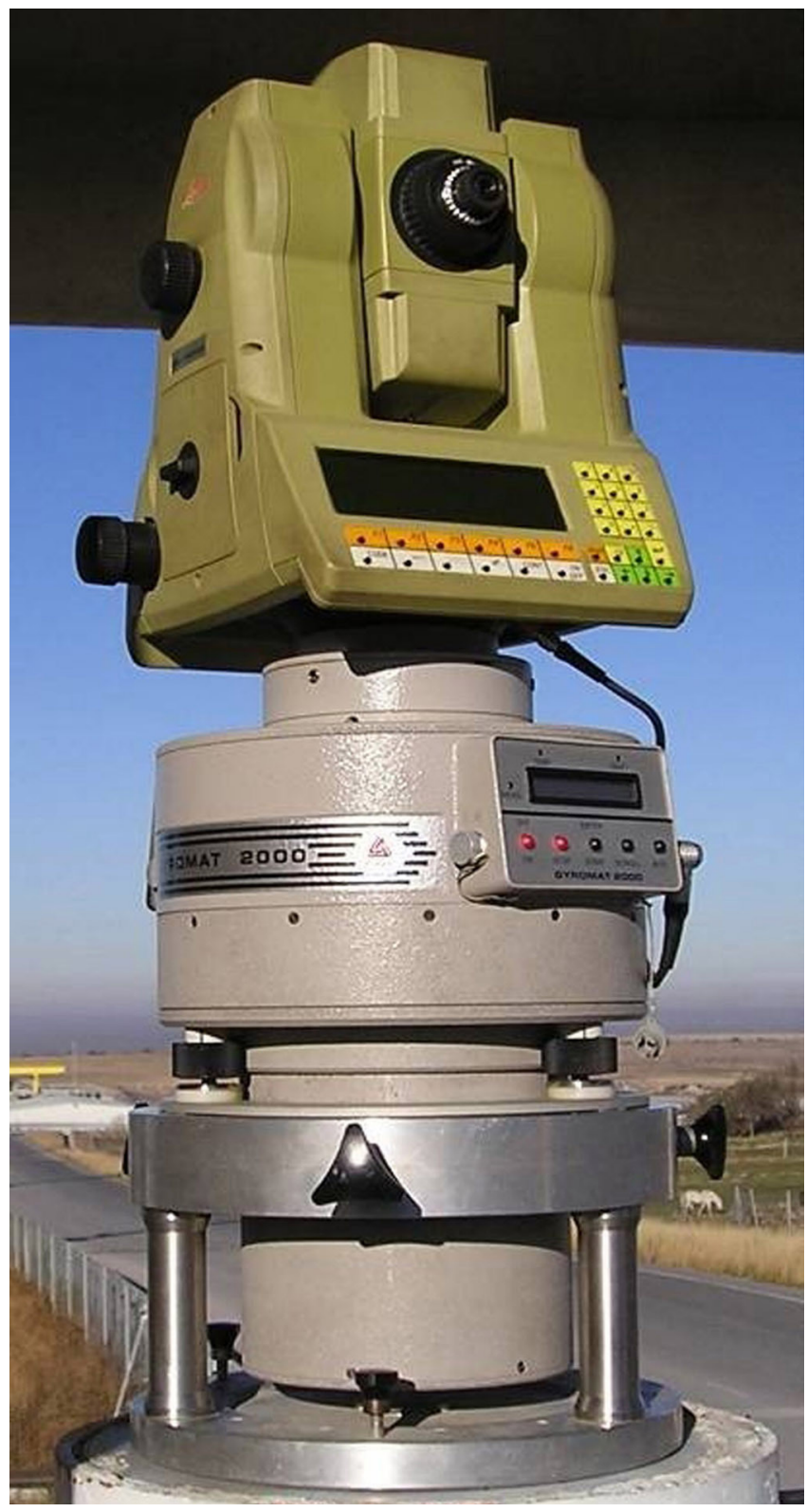

Figura 1. Conjuto de giroteodolito puesto en estación. El elemento central es un giróscopo Gyromat 2000 al que se le ha adaptado, en su parte superior, una estación total robotizada Leica TCA2003, que es la que ofrece la lectura angular del acimut determinado por el giróscopo. El conjunto va montado sobre una bancada que posibilita su puesta en estación sobre el pilar de observación.

ponente es necesario conocer el geoide en la zona de trabajo, por lo que habría que determinarlo o, en su defecto, utilizar un modelo del geoide.

\subsection{Errores en las observaciones}

Los errores en las observaciones son: error angular, error longitudinal, error de estacionamiento y error de puntería por lo que el error total que se produce en una poligonal vendrá dado (15) por la siguiente expresión [1]:

$$
\sigma_{t}=\sqrt{\sigma_{a}^{2}+\sigma_{l}^{2}+\sigma_{e}^{2}+\sigma_{p}^{2}}
$$

Si consideramos una ETR con un error de 2 cc (2 segundos centesimales) y $( \pm 2 \mathrm{~mm}+2 \mathrm{ppm})$, sustituyendo en la expresión [1] obtenemos los errores de cierre en mm debido al error total en función de la longitud de la poligonal y la longitud de los ejes (Tabla 1).

Si comparamos el error angular y el error total, se puede comprobar que las diferencias no superan en ningún caso los 3 $\mathrm{mm}$, por lo que a la vista de la Tabla 2, la calidad de los resultados dependen fundamentalmente de la precisión en la observación angular.

Además de los errores propios de una observación, como se refleja en el apartado anterior, en éste tipo de obras hay que añadir dos fuentes de error como son el de la refracción lateral y el de la desviación de la vertical.

\subsection{Refracción lateral}

El fenómeno de la refracción se presenta al atravesar un haz luminoso diferentes capas de aire de distintas densidades. El error de refracción vertical es un fenómeno perfectamente conocido en el mundo de la Geodesia y de la Topografía, pero el efecto de refracción lateral es un fenómeno menos conocido. Simplificando, la refracción lateral es la misma que la refracción vertical pero actuando en una dirección perpendicular (16).

La solución más satisfactoria para evitar el error lateral sería realizar la poligonal por el centro del túnel pues en él el flujo de aire es laminar, no turbulento y no hay gradiente térmico al ser constantes las condiciones. Pero por lo general el centro del túnel no puede ser utilizado para llevar la poligonal pues suele ser utilizado para el transporte por lo que las poligonales se llevan por los hastiales.

Tabla 1. Error total (en mm).

\begin{tabular}{|c|c|c|c|c|c|c|c|c|}
\hline $\begin{array}{c}\text { EJE (m) / } \\
\text { LONG. (km) }\end{array}$ & $\mathbf{2}$ & $\mathbf{4}$ & $\mathbf{6}$ & $\mathbf{8}$ & $\mathbf{1 0}$ & $\mathbf{1 2}$ & $\mathbf{1 4}$ & $\mathbf{1 6}$ \\
\hline $\mathbf{1 2 5}$ & 17,9 & 44,1 & 78,3 & 118,9 & 165,1 & 216,2 & 271,7 & 331,3 \\
\hline $\mathbf{2 5 0}$ & 13,0 & 31,8 & 56,2 & 85,0 & 117,8 & 154,0 & 193,4 & 235,6 \\
\hline $\mathbf{3 7 5}$ & 12,6 & 27,6 & 46,5 & 73,4 & 98,8 & 126,7 & 163,1 & 195,7 \\
\hline $\mathbf{5 0 0}$ & 9,8 & 23,4 & 40,9 & 61,5 & 84,8 & 110,6 & 138,5 & 168,5 \\
\hline
\end{tabular}

Tabla 2. Diferencias entre el error total y el error angular (en mm).

\begin{tabular}{|c|c|c|c|c|c|c|c|c|}
\hline $\begin{array}{c}\text { EJE (m) / } \\
\text { LONG. (km) }\end{array}$ & $\mathbf{2}$ & $\mathbf{4}$ & $\mathbf{6}$ & $\mathbf{8}$ & $\mathbf{1 0}$ & $\mathbf{1 2}$ & $\mathbf{1 4}$ & $\mathbf{1 6}$ \\
\hline $\mathbf{1 2 5}$ & 2,7 & 2,1 & 1,7 & 1,5 & 1,3 & 1,2 & 1,1 & 1,1 \\
\hline $\mathbf{2 5 0}$ & 1,8 & 1,4 & 1,2 & 1,0 & 0,9 & 0,9 & 0,8 & 0,8 \\
\hline $\mathbf{3 7 5}$ & 1,4 & 1,1 & 1,0 & 0,8 & 0,8 & 0,7 & 0,6 & 0,6 \\
\hline $\mathbf{5 0 0}$ & 1,2 & 1,0 & 0,8 & 0,7 & 0,7 & 0,6 & 0,6 & 0,5 \\
\hline
\end{tabular}

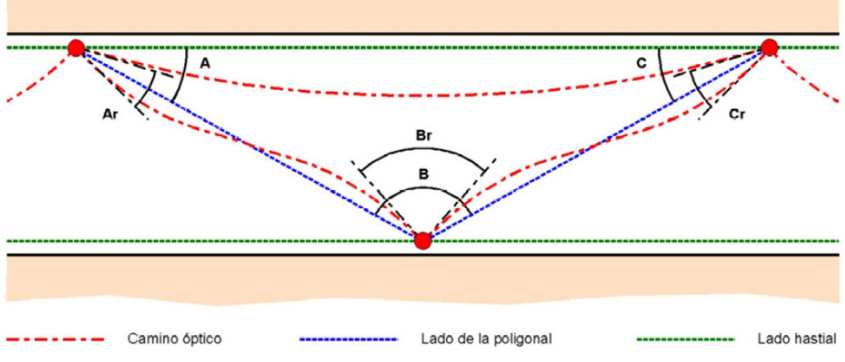

Figura 2. Efecto del error lateral. El camino óptico recto (azul) que debería unir dos puntos de control se ve alterado (rojo) por el efecto de refracción lateral en el interior del túnel. 
La situación alternada hastial - hastial de las estaciones es la única configuración que permite una razonable compensación de la refracción lateral en la transmisión de acimut, a la par que permite su cuantificación. Por supuesto, se supone que el túnel es prácticamente recto y que los gradientes térmicos son simétricos respecto del eje del túnel. Debe considerarse un triángulo aislado (Figura 2) y estudiar la situación bajo condiciones ideales. Antes del planteamiento debe hacerse notar que las desviaciones representadas en la ilustración adjunta, lo mismo podrían ser en el sentido contrario, dependiendo de cómo sean en concreto los gradientes térmicos horizontales. En cualquier caso, las conclusiones del razonamiento no varían.

Los lados cortos A-B y B-C permiten ser observados favorablemente, siendo los destinados a la transmisión angular y al cierre angular del triángulo.

Como puede verse, los ángulos extremos A y C son igualmente afectados por la refracción, convirtiéndose en los equivalentes $\mathrm{Ar}$ y $\mathrm{Cr}$, manteniendo sus valores. El ángulo central si se ve claramente disminuido, convirtiéndose en el Br. El cierre angular del triángulo permite cuantificar el efecto de la refracción. El lado A-C, muy próximo al hastial, es demasiado largo, siendo su única función la citada cuantificación. El error angular debido a la refracción lateral puede ser calculado (17) a partir de la expresión [2]:

$$
\gamma^{\prime \prime}=8 " \frac{P S}{T^{2}}\left(\frac{d T}{d Y}\right)
$$

Si consideramos los ejes de la poligonal iguales y ángulos de $180^{\circ}$ el error debido a la refracción horizontal viene dado (para n ejes).

$$
\Delta Y=n^{2} L^{2} 8^{\prime \prime} \frac{P}{T^{2} \rho^{\prime \prime}}\left(\frac{d T}{d Y}\right) \operatorname{con} \rho^{\prime \prime}=206265
$$

Si utilizamos el giróscopo el error vendrá dado por:

$$
\Delta Y=n L^{2} 8^{\prime \prime} \frac{P}{T^{2} \rho^{\prime \prime}}\left(\frac{d T}{d Y}\right)
$$

Comparando estas expresiones se puede apreciar que la influencia debida al error de refracción lateral utilizando giróscopo en $n$ veces menor que utilizando ETR (18).

En el Eurotúnel fue analizado el problema del efecto de refracción lateral (19) y (20) y mediante la utilización del giroteodolito se mejoró y corrigió dicho error. Fruto de la experiencia en la construcción del túnel del Canal de la Mancha así como de las pruebas realizadas en los túneles de San Gotardo (21), se llegó a las siguientes conclusiones:

1. Huir siempre de visuales dispuestas a menos de 1,5 metros de las paredes del túnel, es decir, se deben evitar visuales paralelas y cercanas a los hastiales.

2. Utilización del giróscopo que minimiza el error debido a la refracción lateral.

\subsection{Error por desviación de la vertical}

En estudios realizados en Nueva Zelanda donde se alcanzan valores de la desviación de la vertical de 30", el error en el ángulo horizontal era de 12" (22).

Las componentes de la desviación de la vertical vienen dador por $(\xi, \eta)$, a partir de estos valores se pueden calcular el valor de la desviación de la vertical y el acimut a partir de las expresiones [5] y [6]:

$$
\begin{gathered}
\theta=\xi " \cos \alpha+\eta " \operatorname{sen} \alpha \\
\alpha=\tan ^{-1}\left(\frac{\eta}{\xi}\right)
\end{gathered}
$$

El error horizontal debido a la desviación de la vertical viene dado por la expresión [7]

$$
\varepsilon=\theta^{\prime \prime} \operatorname{sen}(A-\alpha) \tan V
$$

Donde $A$ es el acimut y $V$ el ángulo vertical

Si las observaciones de las redes interiores se realizan con $V=0$, el error sería cero, es decir la visual debe ser lo más próxima a la horizontal, lo que normalmente ocurre cuando se realizan observaciones en el interior de los túneles. Para $(\xi$, $\eta)=\left(-2,5^{\prime \prime} .2,6^{\prime \prime}\right)$, los valores de a y $\theta$ serían $134^{\circ}$ y $3,6^{\prime \prime}$ respectivamente. Suponiendo $\mathrm{A}=180^{\circ}$ obtendríamos un error de dirección en función del vertical observado (Tabla 3).

Para un túnel de $10 \mathrm{~km}$ de longitud dicho error por desviación de la vertical nos daría un error en el calado del túnel de: 27 , $21,15,10$ y $\mathrm{o} \mathrm{mm}$ respectivamente (23).

\subsection{Cálculo del error de calado de túneles}

Se puede definir el error de calado en un túnel como la diferencia entre las coordenadas del punto extremo del eje teórico del túnel y las coordenadas obtenidas a partir de mediciones geodésicas y topográficas. Se consideran separadamente el error en planimetría y el error en altimetría y se asumen las siguientes hipótesis:

1. La entrada al túnel se produce desde dos bocas opuestas

2. Las coordenadas de los polos de las bocas son conocidas en el sistema de referencia previamente establecido

3. El punto $\mathrm{P}$ está situado en el eje teórico del túnel y en la mitad de dicho túnel

El error se puede descomponer en dos: - $\ell$ error longitudinal y e error transversal, siendo el error más crítico, el cual está motivado por el error angular. Se muestra en la Tabla 4 el máximo error transversal en mm cuando se llevan las poligonales en un túnel desde dos bocas opuestas.

Si consideramos que el error admisible del calado del túnel es de $10 \mathrm{~mm}$ por $\mathrm{km}$ y teniendo en cuenta únicamente el error angular se aprecia que túneles cuya longitud es mayor

\begin{tabular}{|c|c|}
\hline ÁNGULO & ERROR DE DIRECCIÓN \\
\hline $25^{\circ}$ & $1,2 ”$ \\
\hline $20^{\circ}$ & $0,94 ”$ \\
\hline $15^{\circ}$ & $0,69 ”$ \\
\hline $10^{\circ}$ & $0,46 ”$ \\
\hline $5^{\circ}$ & $0,23 ”$ \\
\hline $\mathbf{O}^{\circ}$ & o" \\
\hline
\end{tabular}
de $12 \mathrm{~km}$ y con ejes de 125 metros supera el error admisible. Se puede ver, lógicamente, que cuanto mayor es la longitud del eje de la poligonal menor es el error angular. La geometría de los túneles para trenes de alta velocidad permite vi-

Tabla 3. Error por desviación de la vertical. 
Tabla 4. Error transversal (en mm) con poligonales desde las dos bocas.

\begin{tabular}{|c|c|c|c|c|c|c|c|c|}
\hline $\begin{array}{c}\text { LONG. (km) } \\
\text { / EJE (km) }\end{array}$ & $\mathbf{2}$ & $\mathbf{4}$ & $\mathbf{6}$ & $\mathbf{8}$ & $\mathbf{1 0}$ & $\mathbf{1 2}$ & $\mathbf{1 4}$ & $\mathbf{1 6}$ \\
\hline $\begin{array}{c}\text { LONG. } \\
\text { TOTAL } \\
\text { TUNEL }\end{array}$ & $\mathbf{4}$ & $\mathbf{8}$ & $\mathbf{1 2}$ & $\mathbf{1 6}$ & $\mathbf{2 0}$ & $\mathbf{2 4}$ & $\mathbf{2 8}$ & $\mathbf{3 2}$ \\
\hline $\mathbf{0 , 1 2 5}$ & 25,3 & 62,3 & 110,7 & 168,2 & 233,5 & 305,7 & 384,2 & 468,6 \\
\hline $\mathbf{0 , 2 5 0}$ & 18,4 & 45,0 & 79,4 & 120,3 & 166,6 & 217,8 & 273,5 & 333,2 \\
\hline $\mathbf{0 , 3 7 5}$ & 17,9 & 39,1 & 64,9 & 103,8 & 139,8 & 179,2 & 230,7 & 276,8 \\
\hline $\mathbf{0 , 5 0 0}$ & 13,9 & 33,1 & 57,8 & 86,9 & 119,9 & 156,3 & 195,9 & 238,3 \\
\hline
\end{tabular}

suales de estas longitudes pues el trazado no tiene grandes curvaturas. Pero el inconveniente es que la distancia de 375 metros es insuficiente para las labores cotidianas del guiado de la máquina, hay que cambiar el aparato cuando éste llega al final de la máquina y sólo en ese preciso instante (24). Fruto de las experiencias en túneles de Guadarrama y de las distintas simulaciones realizadas, en túneles de Pajares se realizaron poligonales con longitudes de ejes de 250 metros.

\subsection{Monumentación}

La monumentación de los vértices de la red interior principal es un aspecto importante y depende de factores como son: el diámetro del túnel, localización de los distintos servicios. Las estaciones serán unas ménsulas o plataformas fijas (Figura 3) permanentemente montadas en las paredes del túnel.

En cuanto a la señalización de redes secundarias el avance más significativo en control de túneles ha sido la aparición de un nuevo tipo de punterías conocidos con el nombre de spigots los cuales van montados sobre las paredes o en el techo

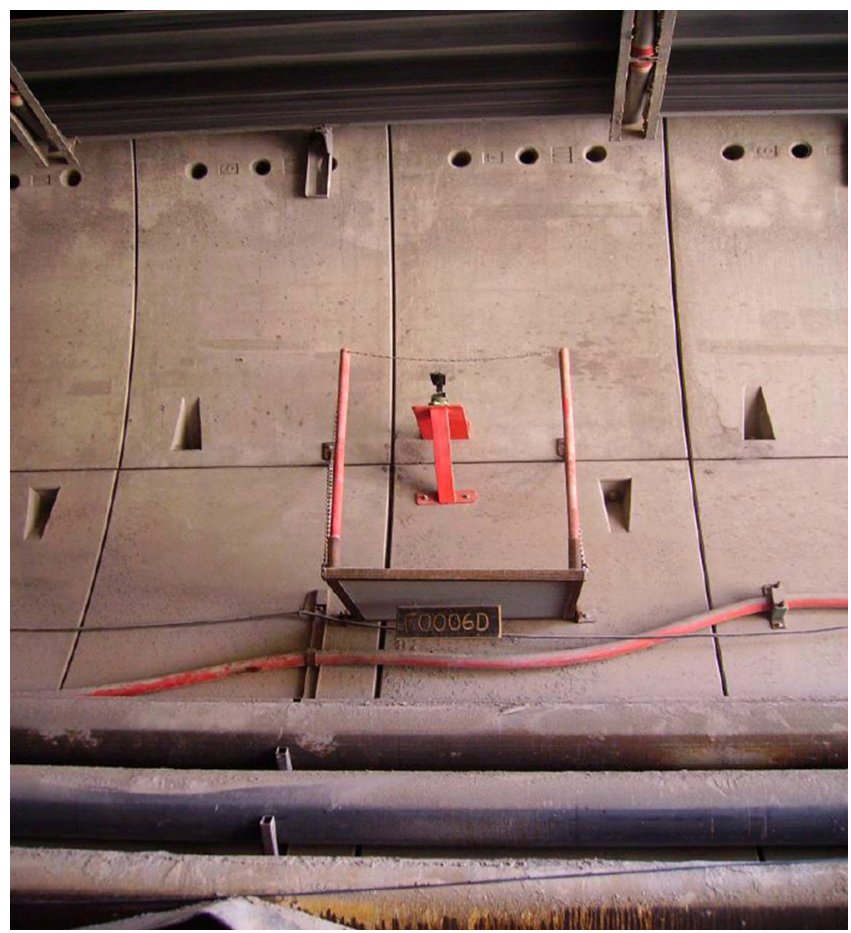

Figura 3. Prisma sobre plataforma. El sistema de plataforma garantiza la repetibilidad en el estacionamiento, tanto de la estación total como del prisma de puntería, durante toda la ejecución del proyecto. del túnel. Son utilizados en el túnel base de San Gotardo (25). Un análisis realizado por (26) muestra que la precisión que se obtiene es comparable a los métodos tradicionales empleados en minerías.

\subsection{Diseño y simulación de redes en el interior de los túneles}

El diseño de las redes interiores debe estar basado en las experiencias previas y en técnicas de simulación. En (27) se reflejan los cuatro pasos para el diseño de una red de control. En cuanto a las simulaciones, se han realizado con el programa Geolab siguiendo la estrategia desarrollada en (28) con el fin de poder analizar y optimizar los distintos diseños que se pueden realizar. Permite trabajar con simulaciones de redes, a fin de evaluar la calidad de la geometría de la red proyectada y elegir la metodología de observación e instrumental necesario para obtener los resultados más precisos posibles.

Para ello se parte de la siguiente hipótesis:

- La precisión exigida en el calado del túnel es de $10 \mathrm{~mm}$ por $\mathrm{km}$ (23).

- Las precisiones de los instrumentos que se han utilizado para realizar la simulación se han obtenido de los distintos equipos que intervienen en las observaciones de túneles de Guadarrama o túneles de Pajares.

- Estación total con 1,5 cc de precisión nominal y 1 mm + 1 ppm.

- Giroteodolito de precisión nominal de 10 cc.

- Se introduce además un error de estacionamiento y puntería de $0,002 \mathrm{~mm}$.

- El túnel es perforado desde los dos extremos.

Se ha realizado un análisis de poligonales por el túnel, entrando al mismo por dos bocas con lados de poligonal de 125, 250 y 375 metros, así como combinaciones de distintas longitudes y poligonales por el eje y cruzadas (zig-zag). Las simulaciones se han realizado en función del número de series de medidas tanto angulares como del giroteodolito, así como para el caso de dos túneles, sensiblemente paralelos.

La observación con giroteodolito se introduce en forma de aspa cada kilómetro y las distancias como dos medidas por eje, pues aumentar el número de series de distancia no mejora significativamente la precisión final del calado del túnel.

De todas las simulaciones realizadas en el estudio, se presentan las las obtenidas para un túnel de $20 \mathrm{~km}$ de longitud con poligonales de 250 metros en zig-zag, considerando la utilización o no del giroteodolito.

En la Figura 4 se representa en el eje de abcisas el número de series angulares realizadas y en el eje de ordenadas las precisiones, al $95 \%$, obtenidas en la simulación en función del número de series.

Como se puede apreciar en la citada gráfica el mínimo número de series que se deberían realizar son: una de giroteodolito y 16 angulares o dos de giroteodolito y dos angulares.

En la Figura 5 se muestran las elipses de error al $95 \%$ para dos poligonales cruzadas de longitudes $10 \mathrm{~km}$ y lados de 250 metros con y sin giroteodolito. 
POLIGONAL CRUZADA, LONGITUD $5 \mathrm{~km}$ Y LADOS DE $250 \mathrm{~m}$

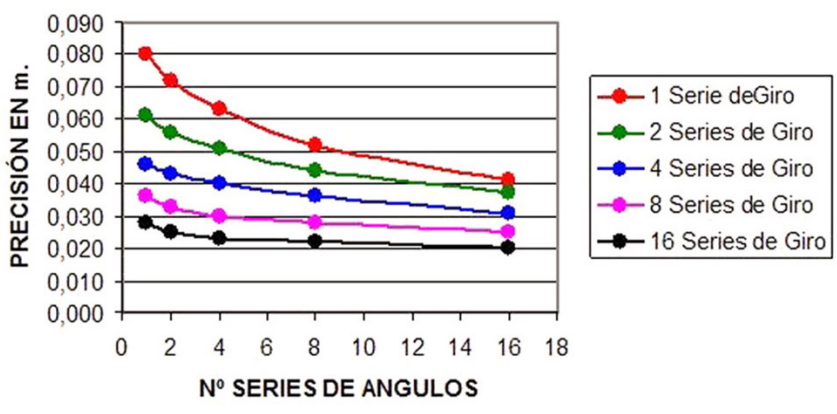

Figura 4. Poligonal cruzada (lados 250 metros) longitud túnel $20 \mathrm{~km}$.

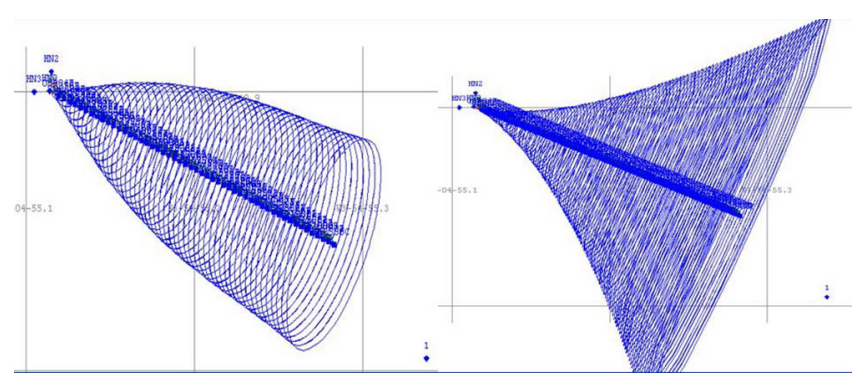

Figura 5. Elipses de error para poligonales cruzadas (lados 250 metros) longitud túnel $20 \mathrm{~km}$ con y sin giroteodolito.

En la Tabla 5 se muestra el resultado de la simulación para túneles de $20 \mathrm{~km}$ con poligonales de $10 \mathrm{~km}$ por cada boca sin utilizar el giroteodolito.

Como se puede apreciar: las poligonales diseñadas por el eje no entrarían en tolerancia sin utilizar el giroteodolito.

En la Figura 6 se puede ver cómo va disminuyendo la precisión de la poligonal llevada por el eje en función de su longitud y de la distancia entre ejes.

En la Tabla 6 se muestran las series angulares y de giroteodolito que se deberían realizar en función de las longitudes de las poligonales (cruzadas y con ejes de 250 metros de longitud) así como las precisiones obtenidas al $95 \%$.

\section{RESULTADOS Y DISCUSIÓN}

A partir de las conclusiones de los trabajos realizados en los Túneles de Guadarrama, y de las simulaciones ante-

Tabla 5. Poligonales sin utilización del giroteodolito (túnel $20 \mathrm{~km}$ ).

\begin{tabular}{|c|c|c|c|}
\hline \multirow[t]{4}{*}{$\begin{array}{l}\text { Poligonal } \\
\text { por el eje }\end{array}$} & Longitud de lados & $\begin{array}{l}\mathrm{N}^{0} \text { series } \\
\text { angulares }\end{array}$ & $\begin{array}{c}\text { Error } \\
\text { de cierre }\end{array}$ \\
\hline & 125 metros & 16 & 0,429 metros \\
\hline & 250 metros & 16 & 0,182 metros \\
\hline & 375 metros & 16 & 0,126 metros \\
\hline \multirow{3}{*}{$\begin{array}{l}\text { Poligonal } \\
\text { cruzada }\end{array}$} & 125 metros & 16 & 0,294 metros \\
\hline & 250 metros & 16 & 0,129 metros \\
\hline & 375 metros & 16 & o,089 metros \\
\hline \multirow{4}{*}{$\begin{array}{l}\text { Poligonal } \\
\text { cruzada }\end{array}$} & 125 y 250 metros & 16 & 0,117 metros \\
\hline & 125 y 375 metros & 16 & o,o84 metros \\
\hline & 250 y 375 metros & 16 & o,o73 metros \\
\hline & 125,250 y 375 metros & 16 & o,o71 metros \\
\hline
\end{tabular}

POLIGONALES SIN GIRO

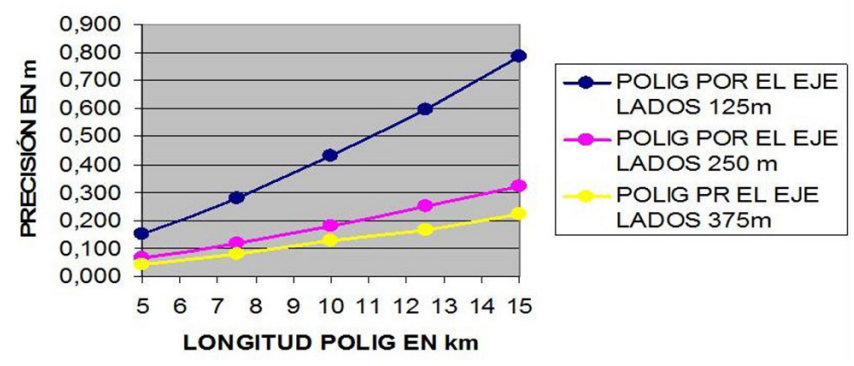

Figura 6. Poligonales por el eje sin giroteodolito.

riormente presentadas se decidió por fijar lados de longitud de 250 metros, cuya distancia da el mayor rendimiento con unos errores angulares perfectamente aceptables para el calado de los túneles. Para evitar el efecto de refracción lateral la observación se ha realizado con visuales cruzadas sobre el eje del túnel de un hastial a otro que minimicen dicho efecto.

El tipo de señalización ha sido el de ménsulas metálicas ancladas a los hastiales del túnel, niveladas y con tornillos 5/8" para estacionamiento de los equipos.

Para el diseño de la observación planimétrica de las redes en el interior de los túneles, la observación presenta tres facetas: medida de distancias, de ángulos y de acimutes giroscópicos.

Las mediciones angulares siempre se han realizado con el programa de «medición de series» de la TCR, con la técnica de vuelta de horizonte. Una vez realizadas las series se calculan in situ los ángulos y distancias medios además de la desviación estándar de la serie y de una medida aislada en ángulos horizontales, verticales y distancias. Si la desviación estándar es mayor de 5 cc se rechaza la serie. Si la desviación estándar entre series es mayor de 5 cc se repiten dos más, rechazándose la mayor y la menor. Si entonces se cumple la tolerancia mencionada, queda aceptada la observación.

Los resultados obtenidos en el tramo Sur del túnel Este (de Pajares), el cual ha sido perforado desde tres frentes (Norte, Centro y Sur) han sido los siguientes:

Observación de 78 vértices con mediciones de 1.073 distancias, 20 acimutes con giroteodolito y 557 ángulos horizontales.

La precisión planimétrica al $95 \%$ obtenida fue de $71 \mathrm{~mm}$ en dicho tramo del túnel cuya longitud es de aproximadamente $10 \mathrm{~km}$.

Tabla 6. Resumen de resultados para poligonales cruzadas con ejes de 250 metros.

\begin{tabular}{|l|c|c|c|c|c|}
\hline $\begin{array}{l}\text { Longitud } \\
\text { Poligonal }\end{array}$ & $\mathbf{5} \mathbf{~ k m}$ & $7,5 \mathbf{~ k m}$ & $\mathbf{1 0} \mathbf{~ k m}$ & $\mathbf{1 2 , 5} \mathbf{~ k m}$ & $\mathbf{1 5} \mathbf{~ k m}$ \\
\hline $\begin{array}{l}\text { no series } \\
\text { giroteodolito }\end{array}$ & 2 & 2 & 2 & 2 & 2 \\
\hline $\begin{array}{l}\mathbf{n}^{\mathbf{o}} \text { series de } \\
\text { ángulos }\end{array}$ & 8 & 4 & 2 & 2 & 2 \\
\hline $\begin{array}{l}\text { Desviaciones } \\
\text { al 95\% }\end{array}$ & $0,044 \mathrm{~m}$ & $0,069 \mathrm{~m}$ & $0,089 \mathrm{~m}$ & $0,101 \mathrm{~m}$ & $0,115 \mathrm{~m}$ \\
\hline
\end{tabular}




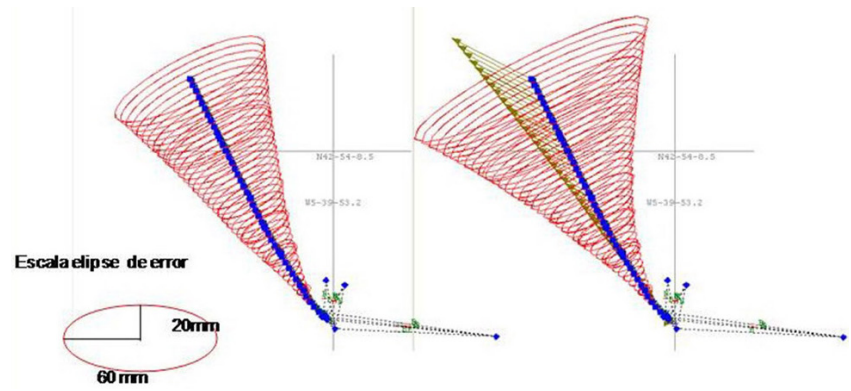

Figura 7. Elipse de error con giroteodolito y sin giroteodolito.

En la Figura 7 se muestran las elipses de error obtenidas con y sin giroteodolito.

Con el programa de cálculo Geolab, de forma similar a (28) y a partir de los datos de observación distintas simulaciones en los tres tramos según lo comentado en el apartado 2.8. Aquí se presentan los resultados obtenidos en el tramo norte cuya longitud aproximada es de $10 \mathrm{~km}$ :

Si se elimina el $67 \%$ de las distancias y el $40 \%$ de ángulos se obtiene una precisión planimétrica al $95 \%$ de $83 \mathrm{~mm}$.

$\mathrm{Al}$ introducir dos series de observaciones de giroteodolito la precisión planimétrica al $95 \%$ es de $67 \mathrm{~mm}$.

Si se eliminan las observaciones del giroteodolito se obtiene una precisión planimétrica al $95 \%$ de $130 \mathrm{~mm}$.

Fruto de la experiencia de las observaciones realizadas, la observación de cada serie angular supone aproximadamente unos 20 minutos de duración lo cual se traduce en una disminución del 40 \% del tiempo dedicado a la observación de la red interior.

\section{Como resumen:}

Con 4 series de ángulos y 2 de giroteodolito se ha obtenido una precisión planimétrica de $67 \mathrm{~mm}$ al $95 \%$ lo que supone un ahorro del tiempo de observación del $25 \%$.

Si se realizan dos series de observaciones angulares y dos series de giroteodolito se obtiene una precisión planimétrica de $73 \mathrm{~mm}$ con un ahorro en el tiempo de observación del 48 \% del total.

Los resultados obtenidos con las observaciones realizadas coinciden con los obtenidos en el apartado de simulaciones realizadas en éste artículo

El hecho de incorporar las observaciones de giróscopo, a pesar de que su precisión sea de 5 a 8 veces peor que la obtenida con una estación total, hace que además de enlazar las redes de los túneles al marco de referencia terrestre materializado en los puntos de control de las respectivas bocas, sea la única comprobación en el interior de los túneles que por ahora se dispone.

El resultado de este «control» interno en el túnel sobre el mismo marco de referencia terrestre conlleva a que esa acumulación de errores, que representan las observaciones de TCR, no supere un cierto umbral, el cual es fijado en función de la capacidad de maniobrado de la tuneladora, además, cualquier sistematismo de orientación inicial que hubiese en las redes del interior de los túneles, se pondrá de manifiesto con la incorporación de estas lecturas de acimutes giroscópicos.

En cuanto a la necesidad de la utilización del giroteodolito, si bien como se muestra en las distintas simulaciones realizadas se podría obviar su uso, es la única metodología de observación que nos permite hoy por hoy realizar comprobaciones antes del calado de los túneles, por lo que consideramos que su uso debe ser obligado cuando se están realizando túneles con longitudes mayores de $15 \mathrm{~km}$.

\section{REFERENCIAS}

(1) Velasco, J., Prieto, J.F., Herrero, T.R. (2014). Metodología de diseño, observación y cálculo de redes exteriores para túneles de gran longitud. Informes de la Construcción, 66(533): e010, http://dx.doi.org/10.3989/ic.13.007.

(2) Torge, W. (2001). Geodesy ( $3^{\text {a }}$ ed.), p. 210. Berlín: Walter de Gruyter

(3) Ghilani, C.D., Wolf, P.R. (2008). Surveying, p. 234. Upper Saddle River (US-NJ): Pearson Education Limited.

(4) Priego, E. (2002). Túneles y Galerías subterráneas, pp. 28-29. Valencia: Ed. Universidad Politécnica de Valencia.

(5) Morlot, J. J., Aguilhon, R., Boitard, J. (1995). Texto de las jornadas técnicas sobre proyecto y construcción del túnel bajo el Canal de La Mancha (Eurotúnel), pp. 63-64. Madrid: Universidad Politécnica de Madrid, Escuela Universitaria de Ingeniería Técnica Topográfica.

(6) Olsson, M., Svennson, R. (1997). Working as a Surrey controller for the Hallandsasen Railway Tunnel. En FIG-Symposium, (pp 33-45).

(7) Korittke, N., Klapperich, H. (1998). Application of high precision gyrotheodolites in tunnelling. (Proceedings Paper). Tunnels and Metropolises, 1(2): 823-827.

(8) Lambrou, E., Pantazis, G. (2004). Accurate Orientation of the Giroscope's calibration system. En FIG Athenes, (pp. 4264). Athenes: FIG.

(9) Lewen, I. (2006). Use of gyrotheodolite in underground control network (Tesis doctoral). Stockholm: Royal Institute of Technology.

(10) Brunner, F.K., Grillmayer, E. (2002). On the temperature dependence of gyroscopic measurements using the GYROMAT 2000. En FIG XXII International Congress. Washington DC, USA.

(11) Gyromat, D. (2000). Operator's Guide, pp. 4-7. Essen: DMT GmbH \& Co. KG.

(12) Navarro, A., Prieto, J.F., Velasco, J. (2009). El guiado de las tuneladoras en los túneles de Pajares. En Miguez, R. (Ed.), Túneles de Pajares, (pp 253-278). Móstoles: ADIF.

(13) Caturla, JL. (1978). Determinación de puntos Laplace, pp. 9-12. Madrid: Instituto Geográfico Nacional.

(14) Sevilla, M.J. (1979). Determinación del acimut de una referencia por observaciones a la estrella polar. Programa de cálculo automático, pp. 7. Madrid: Universidad Complutense de Madrid. 
(15) Ghilani, C.D., Wolf, P.R. (2006). Adjustment Computations Spatial Data Analysis (4 ${ }^{\mathrm{a}}$ ed.), p. 106. Hoboken: John Wiley \& Sons.

(16) Johnston, A. (1998). Tunnel alignment and lateral refraction. Tunnels \& Tunnelling International, 30(3): 58-60.

(17) Blachut, T. J., Chrzanowski, A., Saastamoinen, J. H. (1979). Urban surveying and mapping, pp.93-120. Nueva York: Springer-Verlag,

(18) Chrzanowski, A. (1981). Optimization of the Breakthrough Accuracy in Tunneling Surveys. The Canadian Surveyor, 35(1): 5-16.

(19) Johnston, A. (1991). Lateral refraction in tunnels. Survey review, 31(242): 201-220.

(20) Korittke, N. (1990). Influence of Horizontal Refraction on Traverse Measurements in Tunnels with Small Diameter. En Proceedings of the Second International Workshop on Accelerator Alignment, p. 315.

(21) Ingensand, H., Hennes, M., Böckem, B. (1997). Recent concepts for refraction-free optical measurements in tunnels and vertical shafts. En FIG-Symposium 1997, (pp. 181-195). Copenhagen Road Directorate.

(22) Dymock, P., Hannah, J., Pearson, C. (1999). The second Manapouri Tailrace tunnel: the integration of GPS and conventional surveying in an area of significant deviation of the vertical. The New Zealand Surveyor, 289: 13-18.

(23) Fowler, S. (2006). Design and Preanalysis of Underground Control Networks for Tunnel Construction (Tesis doctoral). Dunedin: University of Otago

(24) Arranz, P. (2005). La topografía en los túneles de Guadarrama. En ADIF (Ed.), Túnel de Guadarrama (pp. 303-346). Madrid: Entorno Gráfico S.L.

(25) Ryf, A., Haag, R., Schätti, I. (2000). AlpTransit Gotthard-Basistunnel: Aktueller Projektstand, ingenieurgeodätische Aspekte. En Schnädelbach, Schilcher (Eds.) Kurs für Ingenieurvermessung, München, XIII. p.5. Stuttgart: Wittwer Verlag.

(26) Shepherd, L., Jarosz, A. (2006). An error analysis of the wall station resection method of underground control traversing, p. 8. Western Australia: Curtin University of technology, Western Australlian School of Mines, Mine Surveying Program kalgoorlie.

(27) Grafarend., E.W. (1974). Optimization of geodetic networks. Bolletino di Geodesia e Science Affini, 33(4): $351-406$.

(28) Regidor Gutiérrez, J., Prieto Morin, J. F., Sanz Mejía, J. M., Quirós Donate, R., Barbadillo Fernández, A. (2001). El Proyecto REGENTE. Topografía y Cartografia, 18(105): 3-11. 